\title{
Mediastinal T Lymphoblastic Lymphoma
}

National Cancer Institute

\section{Source}

National Cancer Institute. Mediastinal T Lymphoblastic Lymphoma. NCI Thesaurus. Code C45740.

A precursor T-lymphoblastic lymphoma that arises from the mediastinum. It usually presents with an anterior mediastinal mass and respiratory distress. It may present acutely with superior vena cava syndrome. It usually affects adolescent and young adult males. It is treated with aggressive chemotherapy and radiation. The prognosis is better in the pediatric patients. 